\title{
Estrategias pasivas de optimización energética de la vivienda social en clima mediterráneo
}

\section{Passive strategies for energy optimisation of social housing in the Mediterranean climate}

$\underline{\text { R. Suárez }}{ }^{(*)}$, J. Fragoso ${ }^{(*)}$

\section{RESUMEN}

Los objetivos prioritarios del Horizonte 2020 han llevado a la actualización del Documento Básico de Ahorro de la Energía del CTE en 2013, introduciendo un nuevo modelo en las exigencias, basado en parámetros tecnológicos y constructivos, asociado a una mayor valoración de las condiciones arquitectónicas del edificio.

Este trabajo pretende analizar la repercusión del nuevo marco normativo en la vivienda social del área mediterránea, en la zona climática B4. Se plantea el análisis energético en un modelo base de bloque lineal entre medianeras al que se incorporan individualmente, distintas estrategias pasivas asociadas a la compacidad, materialidad de la envolvente, control solar, acumulación solar y ventilación, analizando la mejora de la demanda de energía, la calificación energética y el confort interior. La consideración de la orientación, junto con la combinación de la mejora de las prestaciones energéticas de la envolvente térmica, de la tasa de ventilación y de la adecuada protección solar constituyen las principales acciones de mejora energética que permiten conseguir importantes reducciones de la demanda energética, de las emisiones de $\mathrm{CO}_{2}$ y mejora del confort interior.

Palabras clave: Eficiencia energética; Edificios de Energía Casi Nula (EECN); confort; sistemas pasivos; vivienda social.

\section{ABSTRACT}

The main goals of Horizon 2020 have led to the updating of the Basic Document on Energy Saving of the Technical Building Code in 2013. The demands of the new model, based on technological and construction parameters, are associated with a more extensive assessment of the architectural conditions of buildings.

This study aims to analyse the repercussion of the new regulations on Mediterranean social housing in climate zone B4. It proposes energy analysis on a basic model of a single linear block, adding different individual passive strategies relating to compactness, envelope material, solar control, solar accumulation and ventilation, analysing the improvement in energy demand, energy rating and indoor comfort. The main energy improvement actions used take into account orientation as well as the combined improvement in energy performance of the thermal envelope, ventilation rate and suitable solar protection. These lead to major reductions in energy demand and $\mathrm{CO}_{2}$ emissions while improving indoor comfort conditions.

Keywords: Energy efficiency; Nearly Zero Energy Building (NZEB); comfort; passive systems; social housing.

${ }^{(*)}$ IUACC, Escuela Técnica Superior de Arquitectura - Universidad de Sevilla (España) Persona de contacto/Corresponding author: rsuarez@us.es (R. Suárez)

Cómo citar este artículo/Citation: Suárez, R., Fragoso, J. (2016). Estrategias pasivas de optimización energética de la vivienda social en clima mediterráneo. Informes de la Construcción, 68(541): e136, doi: http://dx.doi.org/10.3989/ic.15.050.

Licencia / License: Salvo indicación contraria, todos los contenidos de la edición electrónica de Informes de la Construcción se distribuyen bajo una licencia de uso y distribución Creative Commons Reconocimiento no Comercial 3.o. España (cc-by-nc). 


\section{INTRODUCCIÓN}

La normativa española en materia energética ha ido avanzando en sus exigencias desde la norma básica de edificación, NBE CT-79, que regulaba las condiciones térmicas de los edificios (1). Para ello establecía una variable de transmisión global del edificio, el coeficiente de transmisión térmica global $\left(\mathrm{K}_{\mathrm{G}}\right)$, media ponderada de los coeficientes de transmisión de los elementos opacos en contacto con el exterior, con locales no calefactados, con otros edificios y con el terreno. Este coeficiente $\mathrm{K}_{\mathrm{G}}$ se limitaba en función del factor de forma del edificio, de la zona climática y del tipo de energía utilizada en el sistema de calefacción, no existiendo limitaciones para el periodo de refrigeración, ni valores máximos de transmitancias térmicas de ventanas.

La aprobación del Código Técnico de la Edificación (2), supone una mejora cualitativa y cuantitativa de las prestaciones térmicas del edificio, estableciendo nuevas exigencias para la envolvente del edificio. Por un lado, se exigen valores más restrictivos para las transmitancias térmicas de la parte opaca de los cerramientos y por otro, se establecen limitaciones en los huecos, tanto de la transmitancia térmica como del factor solar modificado, en función del porcentaje de huecos para cada orientación, de la zona climática y de la carga interna (referido al factor solar modificado), suponiendo una primera limitación a la demanda de refrigeración, reduciendo el incremento de cargas térmicas debidas a la radiación solar en el periodo de verano, con respecto a la normativa anterior.

Sin embargo presenta dos claras deficiencias:

- El paso del concepto de coeficiente global $\mathrm{K}_{\mathrm{G}}$ a coeficientes de transferencia de cada elemento constructivo de la envolvente y la posibilidad de justificación mediante un método simplificado, en el que el indicador principal son los coeficientes de transferencia estacionarios, en ausencia de radiación solar, permite elevados consumos energéticos en edificios con elevados valores de compacidad.

- Una metodología de cálculo autorreferente, en el que los edificios residenciales se comparan con unos valores estadísticos derivados del estudio del parque residencial.

De esta manera los indicadores empleados, basados en la limitación de la demanda energética, son inapropiados para alcanzar las exigencias de la Directiva 2002/91/CE, al no proporcionar información cuantitativa alguna sobre el consumo del edificio y no tener ningún mecanismo efectivo para controlar y acotar dicho consumo mínimo.

En este sentido el paso fundamental en la actualización del Documento Básico de Ahorro de Energía (DB HE 2013) (3) consiste en el establecimiento de una limitación al consumo de energía primaria no renovable y una limitación a la demanda energética, buscando con ello la convergencia europea en materia de energía (4). Se trata así de alcanzar el objetivo recogido en la Directiva 2010/31/UE de edificios nuevos de energía casi nula (5).

Esta temática ha sido abordada, tanto a nivel nacional como internacional, desde diferentes perspectivas y en diferentes entornos climáticos. Si bien la mayor parte de los estudios en eficiencia energética están principalmente referidos a zonas climáticas del centro y norte de Europa o de España, existen notables estudios sobre el arco mediterráneo. En cualquier caso se abre la necesidad de un debate sobre un nuevo modelo termodinámico en la organización de los sistemas constructivo-tipológicos, basados en la necesidad de una integración entre arquitectura y técnicas medioambientales (6).

Una de las primeras acciones pasa por la caracterización del comportamiento energético real del parque residencial, que puede ser extrapolado a la edificación de nueva planta, para estimar el ahorro energético asociado principalmente a diferentes estrategias pasivas (7). En el área mediterránea cabe destacar la importancia, para la mejora energética de la vivienda, del empleo combinado de estrategias pasivas como han propuesto diferentes autores.

Jaber y Ajib (8) analizan la influencia de la orientación, el tamaño del hueco y el espesor del aislamiento, cuyo uso combinado permite alcanzar reducciones de un 27,6 \% de la energía anual consumida. Domínguez et al. (9) plantean optimizar la envolvente vertical actuando principalmente en los huecos, aumentando el nivel de aislamiento de éstos en función de la orientación, pudiéndose incorporar menores espesores de aislamiento en la parte opaca, lo que supone obtener reducciones de demanda del orden del $26 \%$. Más allá de la optimización de los valores de transmitancia, la inercia térmica es un factor importante en el comportamiento dinámico (10), en función de la masa y su posición en el cerramiento (11). La envolvente se puede optimizar maximizando la capacidad de acumulación solar incorporando aislamiento por el exterior para mantener el comportamiento dinámico de la envolvente a la vez que se incorpora una barrera térmica y de pérdida de calor como proponen Stazi et al. (12). En un clima mediterráneo, con una importante radiación solar, se mejora el comportamiento energético mediante el empleo de soluciones de control de soleamiento móviles, con mejoras que dependen de la orientación, hora del día, mes del año y sombras arrojadas por la edificación, como han estudiado León et al. (13). Asimismo la incorporación de la ventilación natural en el control térmico de la vivienda, es uno de los aspectos más influyentes como han analizado Sendra et al. (14) y su incorporación como técnica pasiva de refrigeración nocturna (15).

Además existen otros factores, no contemplados en este trabajo como los patrones de uso e intensidad de uso en el área mediterránea y su adaptación a los perfiles socio-económicos (16), la incorporación del papel de los usuarios en el balance energético final (17) (18), que permitan valorar el comportamiento ambiental de las viviendas (19), la posible estimación del consumo energético (20) y su influencia en la condiciones de confort interior (21) (22) junto con la implicaciones de los costes de construcción de cada una de las estrategias pasivas y sus prestaciones energéticas (23). Este trabajo pretende ofrecer una serie de estrategias de actuación, más allá de soluciones constructivas concretas, para la mejora del comportamiento ambiental y energético de la vivienda social de nueva construcción en clima mediterráneo, concretamente en la zona climática B4, adaptándose a los actuales compromisos ambientales y cumplimientos normativos en materia de energía, mediante el diseño pasivo del edificio, buscando con ello la convergencia europea en materia de energía hacia el Edificio de Energía Casi Nula. El empleo de las actuales herramientas informáticas de simulación energética y ambiental permite cuantificar la influencia en el ambiente térmico de las principales estrategias asociadas a la compacidad, la materialidad de la envolvente, el control solar, la acumulación solar y la ventilación, así como la variación en la calificación 
energética, favoreciendo la toma de decisiones en cuanto al diseño de los sistemas pasivos, en busca de un equilibrio entre salubridad, habitabilidad y sostenibilidad.

\section{METODOLOGÍA}

Se desarrolla una metodología que permita conocer los cambios cuantitativos que supone la nueva normativa DB HE 2013 y que pueda ser de aplicación a cualquier modelo tipológico y zona climática. Para ello se define un modelo base que responda a un programa habitual de vivienda social, con unas características constructivas adaptadas al cumplimiento de las exigencias de la anterior normativa, DB HE 2006. A partir del modelo base se incorporan distintas estrategias pasivas de forma individual y se analiza su repercusión energética y ambiental, permitiendo establecer cuáles son las estrategias más significativas para el cumplimiento de las nuevas exigencias.

\subsection{Elección del modelo base}

Inicialmente se plantea partir de un modelo base de simulación utilizado con anterioridad para estudios de similares características. Se estudia la posibilidad de considerar el modelo base utilizado por el Ministerio de Fomento (24) justificativo del cumplimiento de las prescripciones de la Directiva 2010/31/ EU encaminadas a la consecución del objetivo del 20/20/20. Sin embargo se detectan algunas anomalías, principalmente que los modelos tipológicos de bloques de viviendas utilizados presentan unas superficies insuficientes para corresponderse con una distribución real de un programa de vivienda social.
Se plantea alternativamente la posibilidad de adoptar los modelos correspondientes a los tests de calibración para los programas alternativos a LIDER (25), pero al igual que antes presentan una falta de adecuación a un programa de vivienda social, junto con una singularidad en la configuración formal de su envolvente, que dificultan el correcto análisis e influencia de cada una de las diferentes estrategias, que podrían quedar contaminadas por la influencias de las singularidades.

Por todo ello se decide enfocar finalmente el análisis desde un modelo tipológico que responda a un programa básico de vivienda social. El modelo tipológico elegido responde a un edificio plurifamiliar de densidad media, un bloque lineal entre medianeras, con una planta baja con uso comercial y cuatro plantas, dos viviendas estándar de protección oficial de $71,50 \mathrm{~m}^{2}$ por planta, altura libre de 2,6 m, dos orientaciones expuestas, este y oeste, con un $10 \%$ de huecos, dos medianeras, y separación entre zona de día y de noche (Figura 1), compartiendo características con los utilizados en los tests de calibración para los programas alternativos a LIDER.

\subsection{Caracterización de los modelos energéticos de simulación}

Se construyen los modelos base energéticos de simulación que contemplan los datos climáticos de la zona B4, la caracterización de la envolvente, la identificación de los sistemas de acondicionamiento térmico, y las condiciones operaciona-

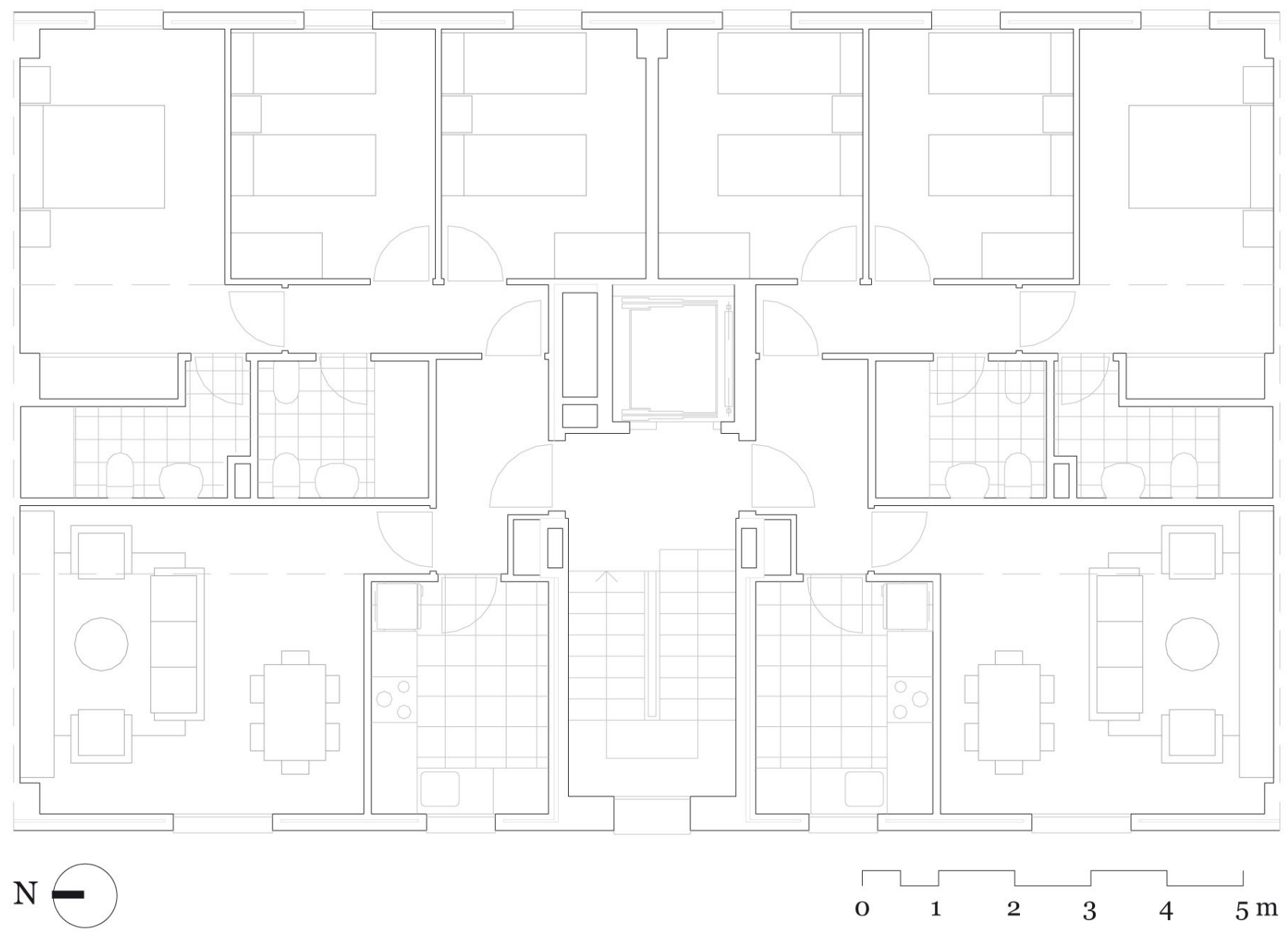

Figura 1. Planta tipo modelo tipológico de simulación base. 
Tabla 1. Caracterización constructiva y térmica de la envolvente del modelo base.

\begin{tabular}{|c|c|c|c|c|c|c|}
\hline \multicolumn{2}{|c|}{ Descripción } & \multicolumn{2}{|c|}{ espesor (m) } & \multicolumn{2}{|c|}{$\mathbf{U}_{\mathbf{m}}\left[\mathbf{W} / \mathbf{m}^{2} \mathbf{k}\right]$} & Masa $\left[\mathrm{kg} / \mathbf{m}^{2}\right]$ \\
\hline \multicolumn{7}{|c|}{ FACHADA (Envolvente exterior): } \\
\hline \multicolumn{2}{|c|}{$\begin{array}{l}\text { Enfoscado de mortero, } \\
1 / 2 \text { pie de ladrillo perforado, } \\
\text { embarrado, cámara de } 4 \mathrm{~cm} \\
\text { sin ventilar y aislamiento } \\
\text { de EPS de } 2 \mathrm{~cm} \\
(\lambda=0,037 \mathrm{~W} / \mathrm{mK}) \text {, tabique } \\
\text { de ladrillo hueco sencillo, } \\
\text { enlucido de yeso. }\end{array}$} & & & & & 187,10 \\
\hline \multicolumn{7}{|c|}{ CUBIERTA (Envolvente exterior): } \\
\hline \multicolumn{2}{|c|}{$\begin{array}{l}\text { Cubierta plana transitable } \\
\text { sobre forjado unidireccional } \\
\text { con entrevigado de } \\
\text { hormigón de } 25 \mathrm{~cm} \text {, } \\
\text { formación de pendiente de } \\
\text { hormigón de áridos ligeros, } \\
\text { lámina impermeabilizante y } \\
\text { aislamiento de XPS de } 4 \mathrm{~cm} \\
(\lambda=0,029 \mathrm{~W} / \mathrm{mK}) \text {. Acabado } \\
\text { exterior con baldosa } \\
\text { cerámica. }\end{array}$} & & & & & 474,50 \\
\hline \multicolumn{7}{|c|}{ FORJADO DE PLANTA TIPO: } \\
\hline \multicolumn{2}{|c|}{$\begin{array}{l}\text { Forjado unidireccional con } \\
\text { entrevigado de hormigón, } \\
\mathrm{m}=381 \mathrm{~kg} / \mathrm{m}^{2}\end{array}$} & \multicolumn{2}{|c|}{0,27} & \multicolumn{2}{|c|}{2,44} & 381,00 \\
\hline \multicolumn{7}{|c|}{ HUECOS (Envolvente exterior): } \\
\hline Descripción & $\begin{array}{l}\mathbf{U}_{\text {marco }} \\
{[\mathbf{W} /} \\
\left.\mathbf{m}^{2} \mathbf{k}\right]\end{array}$ & $\begin{array}{l}\mathbf{U}_{\text {vidrio }} \\
\text { [W/ } \\
\left.\mathbf{m}^{2} \mathbf{k}\right]\end{array}$ & \multicolumn{2}{|c|}{$\begin{array}{c}\mathbf{U}_{\text {hueco }} \\
{\left[\mathbf{W} / \mathbf{m}^{2} \mathbf{k}\right]}\end{array}$} & $\begin{array}{l}\text { Factor } \\
\text { sombra }\end{array}$ & $\begin{array}{c}\text { Factor } \\
\text { solar } \\
\text { modificado }\end{array}$ \\
\hline $\begin{array}{l}\text { Carpintería } \\
\text { de aluminio } \\
\text { (clase 1), vidrio } \\
\text { sencillo } 4 \mathrm{~mm} \text {. }\end{array}$ & 5,70 & 5,70 & & & 0,82 & 0,55 \\
\hline \multicolumn{7}{|c|}{ SIN Protección solar } \\
\hline \multicolumn{7}{|c|}{ VENTILACIÓN: } \\
\hline \multicolumn{2}{|c|}{$\begin{array}{l}\text { Caudales mínimos según } \\
\text { DB HS3. }\end{array}$} & \multicolumn{5}{|c|}{0,9 renov $/ \mathrm{h}$} \\
\hline
\end{tabular}

les de uso, ocupación, iluminación, ventilación, así como las condiciones de confort adaptativo.

Constructivamente los modelos adaptan sus características constructivas a las exigencias mínimas del cumplimiento del DB-HE 2006 presentes en la tabla 2.1, transmitancia térmica máxima, y tabla 2.2 de valores límites de los parámetros característicos medios (Tabla 1). Las condiciones operacionales de ocupación, iluminación y ventilación se ajustan a las indicadas en el apéndice C, perfiles de uso del DB HE1 2013 para uso residencial.

La principal herramienta de simulación utilizada es el software oficial Herramienta Unificada LIDER-CALENER. Sin embargo, las limitaciones de dicho programa a la hora de plantear modelos de comportamiento que incluyen movimientos convectivos del aire interior, tales como espacios de acumulación y amortiguación térmica, así como la necesidad de un análisis más profundo en otros ámbitos, como el confort interior de la vivienda, hace necesaria la utilización de un software alternativo, el programa DesignBuilder v.2.4.2.026 (26), que utiliza el motor de simulación térmica Energyplus, y permite analizar las temperaturas de las superficies para evaluar el confort radiante, y poder obtener las condiciones de temperatura operativa interior en libre evolución en las viviendas.

Este modelo base se adapta a tres configuraciones diferentes:

- Modelo edificio-vivienda. El modelo edificio-vivienda es el modelo base de simulación de estudio y se introduce para su cálculo en la Herramienta Unificada LIDER-CALENER estableciendo distintas variaciones del mismo en función de las estrategias de intervención pasiva. Los resultados obtenidos permiten una valoración global del bloque, mientras que para alguna valoración más pormenorizada se analiza un modelo que corresponde a una vivienda, con dos posiciones diferentes dentro del bloque: bajo cubierta y ubicada en planta intermedia.

- Modelo celda. El modelo celda de estudio mediante la Herramienta Unificada LIDER-CALENER es un modelo aislado con dimensiones $2,70 \times 3,30 \times 2,60 \mathrm{~m}$, que se corresponde con un dormitorio tipo del modelo edificiovivienda, con todos los cerramientos adiabáticos salvo el plano de fachada en el que se dispone una ventana de 
$0,9 \times 0,9 \mathrm{~m}$. Este modelo se utiliza para comprobar la influencia de un importante número de diferentes soluciones de una misma estrategia en un corto periodo de tiempo, seleccionando las más significativas para evaluarlas en el modelo edificio-vivienda.

- Modelo alternativo de vivienda. El modelo individual de vivienda, en sus dos variantes de posición en el bloque, se analiza mediante el programa DesignBuilder para estudiar las condiciones de confort interior, así como aquellas estrategias pasivas cuya simulación no contempla la Herramienta Unificada LIDER-CALENER. El análisis de los resultados obtenidos permite un estudio de las condiciones interiores de las viviendas en cuanto a confort, demanda y temperaturas operativas en libre evolución, más allá de los datos de carácter global del edificio, durante el día más desfavorable de cada estación: 16 de enero y 12 de julio. La evolución de la temperatura operativa interior se ha comparado con las bandas de temperaturas operativas asociadas al confort adaptativo obtenidas a partir de la media de temperatura exterior de los 7 días anteriores al día tipo, con una banda de confort que oscila entre $19,8^{\circ} \mathrm{C}$ a $24,8^{\circ} \mathrm{C}$ en invierno y de $21,7^{\circ} \mathrm{C}$ a $26,7^{\circ} \mathrm{C}$ en verano.

\subsection{Análisis ambiental y energético. Estrategias de acondicionamiento pasivo}

Se realiza el estudio del comportamiento ambiental de las estrategias pasivas, que se agrupan en varios conjuntos, aplicadas al modelo base de vivienda social:

- Estrategia 1: Compacidad. Se ha analizado la influencia del número de plantas, la altura libre y la influencia en el aumento del porcentaje de fachada manteniendo la superficie en planta del modelo base.

- Estrategia 2: Materialidad de la envolvente. Se valora la repercusión de la mejora tanto del aislamiento de la envolvente térmica opaca, fachada y cubierta, como de la caracterización del hueco, material del marco y tipo de vidrios, junto con la influencia de la relación entre hueco y macizo en la fachada.

- Estrategia 3. Control solar. Se realiza un análisis de diferentes sistemas de control solar. El empleo de filtros que modifican el porcentaje de paso de radiación solar en los huecos y la generación de sombra en las cubiertas; la disposición de barreras que impiden la radiación solar en huecos o fachada, como la disposición de vuelos de terrazas, retranqueo de huecos, así como la configuración de la envolvente mediante sistemas ventilados en fachada y cubierta; o el empleo de espacios de amortiguación térmica en fachada o cubierta, espacios no habitables o no calefactados que actúan como colchón térmico.

- Estrategia 4: Acumulación solar. Se analiza el empleo de la acumulación solar en espacios, acristalamiento de terrazas, o elementos constructivos, inercia térmica en fachadas o forjados; que suponen una modificación de los intercambios y flujos de calor entre el espacio interior y el exterior.

- Estrategia 5: Ventilación. Se analiza la influencia de la tasa de ventilación en la demanda de la vivienda debida a la permeabilidad al aire de las carpinterías y la modificación del caudal de ventilación.

Se considera una estrategia transversal, común a todas ellas, que es la orientación, modificando el modelo base en orientación este-oeste a una orientación norte-sur.
Se simulan estas estrategias de forma individual mediante los distintos modelos energéticos y herramientas de cálculo, para los modelos edificio, celda y vivienda. El análisis de los resultados obtenidos nos informa de la relevancia de las distintas estrategias en el cómputo de la demanda de calefacción y refrigeración del edificio, valorando además el porcentaje de mejora respecto al modelo base.

Tras el análisis de conjunto de las diferentes estrategias individuales y su influencia en el balance energético a partir del modelo base, se desarrollan y analizan propuestas de mejora pasiva en base a la combinación de las estrategias más relevantes, para la reducción de la demanda anual y la mejora de la calificación energética así como de las condiciones de confort interior en la vivienda social de la zona B4 en una tipología de bloque lineal.

Para el estudio de las emisiones de $\mathrm{CO}_{2}$ del modelo base y de las diferentes propuestas de estrategias combinadas mediante la herramienta Calener VYP, para obtener la calificación energética del edificio, se ha tenido en cuenta, para todos los modelos, un sistema de agua caliente sanitaria compuesto por calderas de gas natural individuales para cada vivienda de $10 \mathrm{~kW}$ de potencia total y un factor de rendimiento del 0,9. Como contribución solar mínima se ha considerado un $60 \%$, valor de la zona V obtenido de la tabla 2.1 del DBHE4 2013. En todos los modelos se considera que no existen sistemas activos de climatización, por lo que se considera un sistema por defecto de bajo rendimiento compuesto por calefacción por gasóleo con un rendimiento constante de 0,75 y refrigeración por electricidad con un coeficiente de rendimiento de 1,7 , que penaliza la calificación. La calificación energética está referenciada a emisiones de $\mathrm{CO}_{2}$ y no al indicador de consumo anual de energía primaria por lo que mejores calificaciones energéticas no implican necesariamente menor coste de consumo energético o coste total (27).

\section{ANÁLISIS Y VALORACIÓN DE RESULTADOS}

Se valora la influencia de la modificación de las exigencias energéticas tras la entrada en vigor del DB HE 2013 sobre el modelo base, ajustado al cumplimiento exacto del DB HE 2006. Este modelo se encuentra alejado de las actuales exigencias, con una demanda de calefacción de $27,50 \mathrm{kWh} / \mathrm{m}^{2}$ año, frente a la demanda exigida de $15,0 \mathrm{kWh} / \mathrm{m}^{2} \mathrm{año}$, mientras que la demanda de refrigeración es de 15,o $\mathrm{kWh} / \mathrm{m}^{2}$ año, inferior al valor máximo de 20,0 kWh/ $\mathrm{m}^{2}$ año. Estas demandas serán consideradas como demandas de referencia para valorar el potencial de mejora de cada una de las estrategias.

Previo al desarrollo de las distintas estrategias se analiza la influencia de la orientación como variable fundamental del edificio respecto al modelo base, ya que estará presente en el análisis individual del resto de las estrategias. La disposición del modelo base en orientación norte-sur supone una reducción del $6 \%$ en la demanda de calefacción, al recibir mayor radiación solar en la orientación sur, junto con una reducción significativa de la demanda de refrigeración, en torno al $28 \%$, debido a la influencia de la orientación norte, lo que supone una mejora de un $14 \%$ de la demanda total anual.

\section{Estrategia 1. Compacidad}

El aumento de la compacidad debido al aumento de plantas supone una disminución de la demanda del edificio, debido 
Tabla 2. Resumen comparativo de variantes más significativas de las estrategias de Compacidad y de Materialidad de la envolvente.

\begin{tabular}{|c|c|c|c|c|c|c|c|c|c|c|c|c|}
\hline \multirow{2}{*}{$\begin{array}{l}\text { ESTRATEGIA / } \\
\text { VARIABLES }\end{array}$} & \multicolumn{3}{|c|}{ Este } & \multicolumn{3}{|c|}{ Oeste } & \multicolumn{3}{|c|}{ Norte } & \multicolumn{3}{|c|}{ Sur } \\
\hline & cal & ref & total & cal & ref & total & cal & ref & total & cal & ref & total \\
\hline \multicolumn{13}{|c|}{ Estrategia 1: COMPACIDAD } \\
\hline \multicolumn{13}{|c|}{ 1.1.- $N^{\circ}$ de plantas. [MODELO BASE: $b+4$, coef. compacidad: 3,18 ] } \\
\hline $\begin{array}{c}\mathrm{b}+1 \\
\text { [coef. compacidad: } 1,66 \text { ] }\end{array}$ & $52,0 \%$ & $6,0 \%$ & $35,5 \%$ & $52,0 \%$ & $6,0 \%$ & $35,5 \%$ & $57,0 \%$ & $19,0 \%$ & $46,0 \%$ & $57,0 \%$ & $19,0 \%$ & $46,0 \%$ \\
\hline $\begin{array}{c}\mathrm{b}+7 \\
\text { [coef. compacidad: 3,66] }\end{array}$ & $-8,5 \%$ & $-1,0 \%$ & $-6,0 \%$ & $-8,5 \%$ & $-1,0 \%$ & $-6,0 \%$ & $-8,0 \%$ & $-3,0 \%$ & $-7,0 \%$ & $-8,0 \%$ & $-3,0 \%$ & $-7,0 \%$ \\
\hline \multicolumn{13}{|c|}{ 1.2.- Altura libre de planta. [MODELO BASE: $2,6 \mathrm{~m}$ ] } \\
\hline Altura libre: $2,8 \mathrm{~m}$ & $3,0 \%$ & $-1,0 \%$ & $2,0 \%$ & $3,0 \%$ & $-1,0 \%$ & $2,0 \%$ & $4,5 \%$ & $-1,0 \%$ & $3,0 \%$ & $4,5 \%$ & $-1,0 \%$ & $3,0 \%$ \\
\hline \multicolumn{13}{|c|}{ 1.3.- Aumento de superficie de fachada. [MODELO BASE: o \%] } \\
\hline Aumento fachada: $10 \%$ & $2,0 \%$ & $1,0 \%$ & $1,5 \%$ & $2,0 \%$ & $1,0 \%$ & $1,5 \%$ & $2,5 \%$ & $1,5 \%$ & $2,0 \%$ & $2,5 \%$ & $1,5 \%$ & $2,0 \%$ \\
\hline \multicolumn{13}{|c|}{ Estrategia 2: MATERIALIDAD DE LA ENVOLVENTE } \\
\hline \multicolumn{13}{|c|}{ 2.1.- Aislamiento de fachada. [MODELO BASE: $2 \mathrm{~cm}, \mathrm{EPS} \lambda=0,037 \mathrm{~W} / \mathrm{mK}$ ] } \\
\hline $6 \mathrm{~cm} \mathrm{EPS}$ & $-8,0 \%$ & $-2,5 \%$ & $-6,0 \%$ & $-8,5 \%$ & $-2,0 \%$ & $-6,0 \%$ & $-11,0 \%$ & $-1,0 \%$ & $-8,0 \%$ & $-7,5 \%$ & $-3,0 \%$ & $-6,0 \%$ \\
\hline $7 \mathrm{~cm}$ EPS & $-9,0 \%$ & $-3,0 \%$ & $-7,0 \%$ & $-10,0 \%$ & $-2,0 \%$ & $-7,0 \%$ & $-13,0 \%$ & $-1,0 \%$ & $-9,5 \%$ & $-8,5 \%$ & $-3,0 \%$ & $-7,0 \%$ \\
\hline \multicolumn{13}{|c|}{ 2.2.- Aislamiento de cubierta. [MODELO BASE: $4 \mathrm{~cm}, \mathrm{XPS} \lambda=0,029 \mathrm{~W} / \mathrm{mK}]$} \\
\hline $8 \mathrm{~cm}$ XPS & $-3,0 \%$ & $-1,0 \%$ & $-2,5 \%$ & $-3,0 \%$ & $-1,0 \%$ & $-2,5 \%$ & $-3,0 \%$ & $-2,0 \%$ & $-3,0 \%$ & $-3,0 \%$ & $-2,0 \%$ & $-3,0 \%$ \\
\hline $9 \mathrm{~cm}$ XPS & $-4,0 \%$ & $-1,5 \%$ & $-3,0 \%$ & $-4,0 \%$ & $-1,5 \%$ & $-3,0 \%$ & $-4,0 \%$ & $-4,0 \%$ & $-4,0 \%$ & $-4,0 \%$ & $-4,0 \%$ & $-4,0 \%$ \\
\hline $\begin{array}{c}\text { vivienda cubierta: } 8 \mathrm{~cm} \\
\text { XPS } \\
\end{array}$ & $-10,0 \%$ & $-4,0 \%$ & $-8,0 \%$ & $-10,0 \%$ & $-4,0 \%$ & $-8,0 \%$ & $-11,0 \%$ & $-6,0 \%$ & $-9,5 \%$ & $-11,0 \%$ & $-6,0 \%$ & $-9,5 \%$ \\
\hline \multicolumn{13}{|c|}{ 2.3.- Tipo de carpintería. [MODELO BASE: aluminio U: $5,70 \mathrm{~W} / \mathrm{m}^{2} \mathrm{~K}$ ] } \\
\hline $\begin{array}{c}\text { PVC } 2 \text { huecos } \\
{\left[\mathrm{U}: 2,20 \mathrm{~W} / \mathrm{m}^{2} \mathrm{~K}\right]}\end{array}$ & $-2,0 \%$ & $0,0 \%$ & $-1,0 \%$ & $-2,0 \%$ & $0,0 \%$ & $-1,0 \%$ & $-2,5 \%$ & $0,0 \%$ & $-\mathbf{2}, \mathbf{0} \%$ & $-2,5 \%$ & $0,0 \%$ & $-2,0 \%$ \\
\hline \multicolumn{13}{|c|}{ 2.4.- Tipo de vidrio. [MODELO BASE: sencillo U: $5,70 \mathrm{~W} / \mathrm{m}^{2} \mathrm{~K}$, Fs: o,88] } \\
\hline $\begin{array}{l}\text { 4/6/6 [Fs: o,75 } \\
\text { U: } 3,30 \mathrm{~W} / \mathrm{m}^{2} \mathrm{~K} \text { ] }\end{array}$ & $-2,0 \%$ & $-1,0 \%$ & $-2,0 \%$ & $-2,5 \%$ & $-1,0 \%$ & $-2,0 \%$ & $-3,5 \%$ & $-2,0 \%$ & $-3,0 \%$ & $-2,0 \%$ & $-1,0 \%$ & $-1,5 \%$ \\
\hline $\begin{array}{l}\text { 4/16/6 [Fs: } 0,75, \\
\left.\mathrm{U}: 2,80 \mathrm{~W} / \mathrm{m}^{2} \mathrm{~K}\right]\end{array}$ & $-3,0 \%$ & $-1,0 \%$ & $-2,5 \%$ & $-3,5 \%$ & $-1,0 \%$ & $-2,5 \%$ & $-4,0 \%$ & $-1,0 \%$ & $-3,0 \%$ & $-2,5 \%$ & $-1,0 \%$ & $-2,0 \%$ \\
\hline $\begin{array}{c}\text { 4/6/6 } \operatorname{cint}[\text { Fs: } 0,61, \mathrm{U}: \\
\left.2,5 \mathrm{O} \mathrm{W} / \mathrm{m}^{2} \mathrm{~K}\right]\end{array}$ & $-2,0 \%$ & $-3,5 \%$ & $-2,5 \%$ & $-3,0 \%$ & $-3,5 \%$ & $-3,0 \%$ & $-4,0 \%$ & $-2,0 \%$ & $-3,5 \%$ & $0,0 \%$ & $-3,0 \%$ & $-1,0 \%$ \\
\hline \multicolumn{13}{|c|}{ 2.5.- Porcentaje de huecos de fachada. [MODELO BASE: $10 \%$ ] } \\
\hline $30 \%$ & $-2,0 \%$ & $48,5 \%$ & $8,5 \%$ & $2,0 \%$ & $61,5 \%$ & $14,0 \%$ & $8,0 \%$ & $35,0 \%$ & $12,0 \%$ & $-19,0 \%$ & $48,5 \%$ & $-3,5 \%$ \\
\hline \multicolumn{13}{|c|}{ 2.6.- Forma de huecos. [MODELO BASE: 1 a 1] } \\
\hline 1 a 2 horizontal & $0,5 \%$ & $-0,5 \%$ & $0,5 \%$ & $0,5 \%$ & $-0,5 \%$ & $0,5 \%$ & $0,5 \%$ & $1,0 \%$ & $0,5 \%$ & $1,0 \%$ & $-1,0 \%$ & $0,5 \%$ \\
\hline 1 a 2 vertical & $1,0 \%$ & $0,5 \%$ & $1,0 \%$ & $1,0 \%$ & $0,5 \%$ & $1,0 \%$ & $0,5 \%$ & $0,5 \%$ & $0,5 \%$ & $1,5 \%$ & $0,5 \%$ & $1,0 \%$ \\
\hline
\end{tabular}

principalmente a la influencia de la superficie de cubierta en la demanda del bloque, como queda de manifiesto cuando la altura del bloque disminuye hasta $\mathrm{PB}+1$ con un aumento de la demanda total del $46 \%$ en la orientación norte-sur (Tabla 2). La influencia sobre la demanda de la modificación de la compacidad asociada al aumento de la altura libre y del aumento de superficie de fachada es muy escaso en ambos casos, con variaciones del orden del 2-3 \% de la demanda total.

\section{Estrategia 2. Materialidad de la envolvente}

La mejora de transmitancia térmica del cerramiento vertical, debido al aumento de espesor del aislamiento, supone una mejora energética, principalmente asociada a la demanda de calefacción, del orden del $13 \%$ en la orientación norte, hasta un espesor de unos $7 \mathrm{~cm}$, espesor a partir del cual se atenúa la influencia del espesor sobre la demanda. Aplicado el aumento de espesor del aislamiento a la cubierta, se obtiene una escasa mejora del 3-4 \% de la demanda total en el modelo bloque, para un espesor de 8-9 cm de poliestireno extrusionado.
Sin embargo si se analiza el modelo vivienda de cubierta, este porcentaje de mejora se duplica (Tabla 2).

En la configuración de la ventana, la influencia del marco en el conjunto es escasa, mientras que la mejora de las características del vidrio, bien con un aumento de la cámara de aire, con soluciones de vidrio de 4/16/6 con valores de transmitancia de $2,8 \mathrm{~W} / \mathrm{m}^{2} \mathrm{~K}$, o con el empleo de vidrios bajo emisivos en soluciones estándar 4/6/6, con valores de transmitancia de $2,5 \mathrm{~W} / \mathrm{m}^{2} \mathrm{~K}$ y factor solar de 0,61 , supone mejoras de demanda total del orden de un 2,5-3,5\%, siendo más significativa su influencia en la demanda de calefacción en la orientación norte.

El aumento de la superficie de hueco supone un incremento de la demanda total, debido principalmente al aumento de la demanda de refrigeración del orden del 35-62 \% para un porcentaje de huecos del $30 \%$, excepto en la orientación sur, donde este aumento de la demanda de refrigeración queda compensado con la mejora de la demanda de calefacción, lo que supone una mejora de la demanda total del orden del 
3,5\%. La forma del hueco apenas tiene influencia cuando los porcentajes de hueco no son importantes.

\section{Estrategia 3. Control solar}

La disposición de filtros para el control de soleamiento en huecos con protecciones solares fijas supone en general una reducción de la demanda de refrigeración, en torno a un $6 \%$ para orientaciones este y oeste con un $50 \%$ de protección al paso de la radiación solar, pero se producen incrementos importantes de la demanda de calefacción de hasta un $13 \%$ para orientación sur, lo que supone en general incrementos en la demanda total de hasta un $8 \%$ en orientación sur con dicha protección. Por tanto, la disposición de protecciones solares móviles con un $100 \%$ de protección al paso de la radiación solar que permanezcan cerradas en verano y abiertas en invierno permitirían obtener reducciones de la demanda de refrigeración en torno a un 28 \% en orientación este-oeste y un $5 \%$ en orientación norte-sur, lo que se reflejaría en mejoras de demanda total de hasta un $12 \%$ en orientación este-oeste y un $2 \%$ en orientación norte-sur (Tabla 3).

El empleo de barreras que impiden la radiación solar, tanto en el hueco como en el elemento opaco, supone una reducción del aporte solar en régimen de invierno que generalmente no compensa las mejoras obtenidas en verano, por lo que soluciones puntuales como terrazas voladas, tienen escasa incidencia en la demanda total; incluso el retranqueo de huecos y la disposición de fachadas ventiladas pueden llegar a penalizar la demanda total si la demanda de calefacción es muy superior a la de refrigeración.

El empleo de espacios de amortiguación térmica en fachada supone una reducción principalmente de la demanda de calefacción, pero también de refrigeración, con una reducción de la demanda total que va desde un $3 \%$ en la orientación sur hasta un $5 \%$ en la norte para una disposición de espacios de amortiguación que ocupan un 35 \% de la superficie de fachada.

La disposición de sistemas de protección solar asociados a la cubierta no suponen una reducción significativa de la demanda total del bloque, pero si afectan a la demanda total de la vivienda de cubierta, que se reduce entre un 3-4 \% por la disposición de espacios adosados, generación de sombras o configuración de cubiertas ventiladas, soluciones que reducen el flujo de calor debido a la radiación solar en verano y por lo tanto reducen significativamente la demanda de refrigeración entre un 8-9\%.

\section{Estrategia 4. Acumuladores solares}

El empleo de soluciones de acumulación solar, como el acristalamiento de las terrazas supone una mejora en régimen de invierno, con una reducción de la demanda de calefacción de un $3 \%$, escasa debido al efecto de protección solar que presenta, por lo que éstos deben tener un escaso vuelo, del orden de $0,5 \mathrm{~m}$, que se corresponde con el modelo tradicional. Para evitar el recalentamiento de estos espacios en verano, debe permitirse su correcta ventilación y disponer de un sistema de protección solar (Tabla 3).

El incremento de inercia térmica en fachada, con el aumento de peso y la disposición de aislamiento por el exterior, supone una reducción de la demanda total anual en torno a un $1 \%$.
Sin embargo, si el almacenamiento de calor se realiza en los forjados aumenta la reducción de la demanda total hasta un $5,5 \%$, con un forjado de una masa del orden de $750 \mathrm{~kg} / \mathrm{m}^{2}$.

\section{Estrategia 5. Ventilación}

La reducción de infiltraciones disponiendo carpinterías de mayor estanquidad, produce una importante mejora del orden del $43 \%$ de la demanda de calefacción, y de un $15 \%$ de la demanda de refrigeración en la orientación norte, para una carpintería clase 3 (Tabla 3 ).

Del mismo modo la reducción de la tasa de ventilación supone importantes mejoras. Frente a las actuales soluciones planteadas por el DB HS3, que suponen en el modelo base una tasa de $0,9 \mathrm{ren} / \mathrm{h}$, soluciones más eficientes de ventilación, con valores de $0,30 \mathrm{ren} / \mathrm{h}$ como se exige en el decreto 24/5/2006 francés (28), suponen una reducción de hasta un $38 \%$ de la demanda total, manteniendo las condiciones mínimas de salubridad.

\section{PROPUESTA DE ESTRATEGIAS DE CONJUNTO}

A partir del análisis de sensibilidad de forma individual sobre la demanda de cada estrategia sobre el modelo base, se realiza una serie de propuestas combinadas de diferentes estrategias para reducir la demanda, mejorar la calificación energética y las condiciones de confort interior. Sobre estas propuestas combinadas se realiza un análisis de la variación de demanda respecto del modelo base edificio-vivienda mediante la $\mathrm{He}-$ rramienta Unificada LIDER-Calener, así como un estudio de emisiones de $\mathrm{CO}_{2}$ mediante la herramienta Calener VYP con la que se obtiene la calificación energética del edificio. Paralelamente se realiza un estudio de la temperatura interior en libre evolución para el análisis del confort térmico a partir del modelo de vivienda introducido en el programa DesignBuilder.

Para la definición de las estrategias combinadas se busca introducir acciones de mejora energética capaces de aplicarse de forma genérica a una edificación de vivienda social, evitándose soluciones más particulares, ya que uno de los objetivos de este trabajo es mostrar la posibilidad de acceder a importantes reducciones de la demanda energética con soluciones «accesibles» para la vivienda social.

Se propone en primer lugar una propuesta básica (M), de mínimos asociados a los elementos constructivos, sobre el modelo base, en la que se ajustan los límites de aislamientos de los diferentes elementos constructivos de la envolvente a valores optimizados: fachada, cubierta, carpintería y vidrios. Tras el análisis de las estrategias, se ha comprobado que más allá de la elección de la tipología de cerramiento y valor de transmitancia, es más influyente en el balance energético la inercia térmica y el control de las tasa de infiltraciones. Por lo que se buscan soluciones constructivas que optimicen la influencia de la inercia, principalmente en forjado y con menor entidad en fachada, y carpinterías con una baja tasa de infiltraciones (Tabla 4).

Una vez optimizado el aislamiento de los cerramientos y con una adecuada estanquidad al aire, se complementa la propuesta básica (M) con una optimización de la ventilación (MV), mediante un sistema de ventilación mecánica controlada, optando como referencia con los valores del decreto francés 24/05/2006 (Tabla 4). 
Tabla 3. Resumen comparativo de variantes más significativas de las estrategias de Control solar, Acumuladores solares y Ventilación.

\begin{tabular}{|c|c|c|c|c|c|c|c|c|c|c|c|c|}
\hline \multirow{2}{*}{$\begin{array}{l}\text { ESTRATEGIA / } \\
\text { VARIABLES }\end{array}$} & \multicolumn{3}{|c|}{ Este } & \multicolumn{3}{|c|}{ Oeste } & \multicolumn{3}{|c|}{ Norte } & \multicolumn{3}{|c|}{ Sur } \\
\hline & cal & ref & total & cal & ref & total & cal & ref & total & cal & ref & total \\
\hline \multicolumn{13}{|c|}{ Estrategia 3: CONTROL SOLAR } \\
\hline \multicolumn{13}{|c|}{ 3.1.- Protecciones solares fijas. [MODELO BASE: $100 \%$ apertura] } \\
\hline $\begin{array}{c}50 \% \text { paso } \\
\text { soleamiento }\end{array}$ & $5,0 \%$ & $-6,0 \%$ & $1,0 \%$ & $3,0 \%$ & $-6,0 \%$ & $\mathbf{0 , 0} \%$ & $0,0 \%$ & $0,5 \%$ & $\mathbf{0 , 0} \%$ & $13,0 \%$ & $-5,0 \%$ & $8,0 \%$ \\
\hline $\begin{array}{c}100 \% \text { paso invierno } \\
\text { - o \% verano }\end{array}$ & $0,0 \%$ & $-11,5 \%$ & $-4,0 \%$ & $0,0 \%$ & $-11,5 \%$ & $-4,0 \%$ & $0,0 \%$ & $1,5 \%$ & $0,5 \%$ & $0,0 \%$ & $-9,0 \%$ & $-2,5 \%$ \\
\hline \multicolumn{13}{|c|}{ 3.2.- Altura elemento sombra cubierta. [MODELO BASE: sin elemento] } \\
\hline $\mathrm{h}=2,5 \mathrm{~m}$ & $-1,0 \%$ & $-2,5 \%$ & $-1,5 \%$ & $-1,0 \%$ & $-2,5 \%$ & $-1,5 \%$ & $-0,5 \%$ & $-6,5 \%$ & $-1,5 \%$ & $-0,5 \%$ & $-6,5 \%$ & $-1,5 \%$ \\
\hline $\begin{array}{c}\text { vivienda cubierta } \\
\mathrm{h}=2,5 \mathrm{~m}\end{array}$ & $-1,5 \%$ & $-8,5 \%$ & $-4,0 \%$ & $-3,0 \%$ & $-8,0 \%$ & $-4,5 \%$ & $-1,5 \%$ & $-9,0 \%$ & $-3,5 \%$ & $-2,0 \%$ & $-9,0 \%$ & $-4,0 \%$ \\
\hline \multicolumn{13}{|c|}{ 3.3.- Porcentaje de protección solar cubierta. [MODELO BASE: o \% sin elemento] } \\
\hline $50 \% \mathrm{~h}=2,5 \mathrm{~m}$ & $-1,0 \%$ & $-2,0 \%$ & $-1,5 \%$ & $-1,0 \%$ & $-2,0 \%$ & $-1,5 \%$ & $0,0 \%$ & $-6,0 \%$ & $-2,0 \%$ & $0,0 \%$ & $-6,0 \%$ & $-2,0 \%$ \\
\hline $\begin{array}{c}\text { vivienda cubierta } \\
50 \% \mathrm{~h}=2,5 \mathrm{~m}\end{array}$ & $-1,0 \%$ & $-6,5 \%$ & $-3,0 \%$ & $-5,0 \%$ & $-6,0 \%$ & $-5,0 \%$ & $-3,5 \%$ & $-9,0 \%$ & $-5,0 \%$ & $-4,0 \%$ & $-9,0 \%$ & $-5,0 \%$ \\
\hline \multicolumn{13}{|c|}{ 3.4.- Terrazas vuelos [MODELO BASE: sin vuelo] } \\
\hline $\begin{array}{c}\text { vuelo }=1,5 \mathrm{~m}(25 \% \\
\text { longitud fachada) }\end{array}$ & $-1,5 \%$ & $-1,0 \%$ & $-1,0 \%$ & $-1,0 \%$ & $-1,0 \%$ & $-1,0 \%$ & $-1,0 \%$ & $1,5 \%$ & $-0,5 \%$ & $-1,0 \%$ & $-0,5 \%$ & $-1,0 \%$ \\
\hline \multicolumn{13}{|c|}{ 3.5.- Retranqueo de huecos [MODELO BASE: o,22m] } \\
\hline retranqueo $=0,50 \mathrm{~m}$ & $4,5 \%$ & $-3,0 \%$ & $2,0 \%$ & $3,5 \%$ & $-3,5 \%$ & $\mathbf{1 , 0} \%$ & $0,0 \%$ & $0,0 \%$ & $\mathbf{0 , 0} \%$ & $8,0 \%$ & $-2,5 \%$ & $5,0 \%$ \\
\hline \multicolumn{13}{|c|}{ 3.7.- Fachada ventilada [MODELO BASE: no ventilada] } \\
\hline ventilada & $7,0 \%$ & $-8,5 \%$ & $1,5 \%$ & $6,0 \%$ & $-7,0 \%$ & $\mathbf{1 , 5} \%$ & $4,0 \%$ & $-4,5 \%$ & $1,5 \%$ & $10,0 \%$ & $-11,0 \%$ & $4,0 \%$ \\
\hline \multicolumn{13}{|c|}{ 3.8.- Cubierta ventilada [MODELO BASE: no ventilada] } \\
\hline ventilada & $-3,0 \%$ & $-1,0 \%$ & $-1,5 \%$ & $-3,0 \%$ & $-1,0 \%$ & $-1,5 \%$ & $-7,0 \%$ & $0,5 \%$ & $-1,5 \%$ & $-7,0 \%$ & $0,5 \%$ & $-1,5 \%$ \\
\hline $\begin{array}{l}\text { vivienda cubierta } \\
\text { ventilada }\end{array}$ & $-1,5 \%$ & $-8,5 \%$ & $-4,0 \%$ & $-1,5 \%$ & $-8,5 \%$ & $-4,0 \%$ & $-1,5 \%$ & $-9,5 \%$ & $-4,0 \%$ & $-1,5 \%$ & $-9,5 \%$ & $-4,0 \%$ \\
\hline
\end{tabular}

3.9.- Amortiguación térmica: Espacios adosados fachada [MODELO BASE: sin]

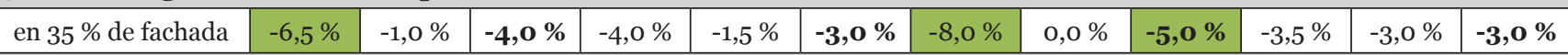
3.10.- Amortiguación térmica: Espacios adosados a cubierta. [MODELO BASE VIVIENDA DE CUBIERTA: sin]

\begin{tabular}{|c|c|c|c|c|c|c|c|c|c|c|c|c|}
\hline $\begin{array}{c}100 \% \text { cubierta viv. } \\
\text { Ocupada }\end{array}$ & $4,0 \%$ & $3,0 \%$ & $3,5 \%$ & $4,0 \%$ & $3,0 \%$ & $3,5 \%$ & $4,0 \%$ & $4,0 \%$ & $4,0 \%$ & $4,0 \%$ & $4,0 \%$ & $4,0 \%$ \\
\hline
\end{tabular}

\section{Estrategia 4: ACUMULADORES SOLARES}

4.1.- Acristalamiento en terrazas. [MODELO BASE: sin terrazas]

\begin{tabular}{|c|c|c|c|c|c|c|c|c|c|c|c|c|}
\hline $\begin{array}{c}\text { vuelo }=0,5 \mathrm{~m} \\
(10 \% \text { fachada })\end{array}$ & $-2,5 \%$ & $0,5 \%$ & $-1,5 \%$ & $-3,0 \%$ & $0,5 \%$ & $-1,5 \%$ & $-3,0 \%$ & $0,5 \%$ & $-1,5 \%$ & $-3,0 \%$ & $2,0 \%$ & $-1,0 \%$ \\
\hline $\begin{array}{c}\text { vuelo = 1,5 m } \\
\text { (10 \% fachada) }\end{array}$ & $-2,5 \%$ & $1,0 \%$ & $-1,0 \%$ & $-2,5 \%$ & $1,0 \%$ & $-1,0 \%$ & $-2,5 \%$ & $1,5 \%$ & $-1,0 \%$ & $-2,5 \%$ & $2,5 \%$ & $-0,5 \%$ \\
\hline
\end{tabular}

4.2.- Inercia térmica: Forjados. [MODELO BASE: FU bovedilla hormigón o,25m, masa: 332,5 kg/m²]

\begin{tabular}{|c|c|c|c|c|c|c|c|c|c|c|c|c|}
\hline $\begin{array}{c}\operatorname{losa} 0,25 \mathrm{~m} \\
{\left[\mathrm{masa}: 625 \mathrm{~kg} / \mathrm{m}^{2}\right]}\end{array}$ & $-2,5 \%$ & $-4,0 \%$ & $-3,0 \%$ & $-2,5 \%$ & $-4,0 \%$ & $-3,0 \%$ & $-2,5 \%$ & $-7,5 \%$ & $-4,0 \%$ & $-2,5 \%$ & $-7,5 \%$ & $-4,0 \%$ \\
\hline $\begin{array}{c}\text { losa } 0,30 \mathrm{~m} \\
{\left[\mathrm{masa}: 750 \mathrm{~kg} / \mathrm{m}^{2}\right]}\end{array}$ & $-3,5 \%$ & $-7,0 \%$ & $-5,0 \%$ & $-3,5 \%$ & $-7,0 \%$ & $-5,0 \%$ & $-4,0 \%$ & $-9,5 \%$ & $-5,5 \%$ & $-4,0 \%$ & $-9,5 \%$ & $-5,5 \%$ \\
\hline
\end{tabular}

4.3.- Inercia térmica: Fachadas. [MODELO BASE: citara + aislamiento + cámara + tabique $\mathrm{U}=\mathbf{0 . 8 2} \mathrm{W} / \mathrm{m}^{2} \mathrm{~K}$ ]

\begin{tabular}{|c|c|c|c|c|c|c|c|c|c|c|c|c|}
\hline $\begin{array}{l}\text { aislamiento exterior } \\
\quad+1 \text { pie ladrillo } \\
{\left[\mathrm{U}=0,82 \mathrm{~W} / \mathrm{m}^{2} \mathrm{~K}\right]}\end{array}$ & $-0,5 \%$ & $-1,5 \%$ & $-1,0 \%$ & $-0,5 \%$ & $-1,0 \%$ & $-0,5 \%$ & $-0,5 \%$ & $-1,5 \%$ & $-1,0 \%$ & $-1,0 \%$ & $-1,5 \%$ & $-1,0 \%$ \\
\hline
\end{tabular}

\section{Estrategia 5: VENTILACIÓN}

5.1.- Permeabilidad al aire de carpinterías [MODELO BASE: clase 1]

\begin{tabular}{|c|c|c|c|c|c|c|c|c|c|c|c|c|}
\hline clase 3 & $-35,0 \%$ & $-9,0 \%$ & $-26,0 \%$ & $-35,0 \%$ & $-9,0 \%$ & $-26,0 \%$ & $-43,0 \%$ & $-16,0 \%$ & $-35,0 \%$ & $-43,0 \%$ & $-16,0 \%$ & $-35,0 \%$ \\
\hline clase 4 & $-38,0 \%$ & $-10,0 \%$ & $-28,0 \%$ & $-38,0 \%$ & $-10,0 \%$ & $-28,0 \%$ & $-46,0 \%$ & $-17,0 \%$ & $-37,0 \%$ & $-46,0 \%$ & $-17,0 \%$ & $-37,0 \%$ \\
\hline \multicolumn{13}{|c|}{ 5.2.- Tasa de ventilación. [MODELO BASE: DB HS3 o,9 ren/h] } \\
\hline $\mathrm{o}, 3 \mathrm{ren} / \mathrm{h}$ & $-38,5 \%$ & $-10,0 \%$ & $-28,5 \%$ & $-39,0 \%$ & $-10,5 \%$ & $-28,5 \%$ & $-47,0 \%$ & $-17,0 \%$ & $-38,0 \%$ & $-46,0 \%$ & $-17,0 \%$ & $-37,5 \%$ \\
\hline
\end{tabular}


Tabla 4. Definición de propuestas combinadas de estrategias.

\begin{tabular}{|c|c|c|c|}
\hline \multicolumn{4}{|c|}{ PROPUESTA BÁSICA (M) } \\
\hline \multirow{8}{*}{$\mathbf{M}$} & $\begin{array}{l}\text { Aislamiento de } \\
\text { fachada }\end{array}$ & $7 \mathrm{~cm}$ & $\operatorname{EPS} \lambda=0,037 \mathrm{~W} / \mathrm{mK}$ \\
\hline & $\begin{array}{l}\text { Aislamiento de } \\
\text { cubierta }\end{array}$ & $8 \mathrm{~cm}$ & $\mathrm{XPS} \lambda=0,029 \mathrm{~W} / \mathrm{mK}$ \\
\hline & \multirow{2}{*}{ Carpintería } & \multicolumn{2}{|c|}{$\mathrm{PVC} 2 \mathrm{huecos} \mathrm{U}=2,20 \mathrm{~W} / \mathrm{m}$} \\
\hline & & \multicolumn{2}{|c|}{ Permeabilidad al aire: Clase 3} \\
\hline & \multirow[t]{2}{*}{ Vidrio } & $\begin{array}{l}\text { Este } \\
\text { Oeste } \\
\text { Sur }\end{array}$ & $\begin{array}{l}4 / 6 / 6 \mathrm{U}=3,30 \mathrm{~W} / \mathrm{m}^{2} \mathrm{~K} \\
\mathrm{Fs}=0,75\end{array}$ \\
\hline & & Norte & $\begin{array}{l}4 / 6 / 6 \varepsilon \text { int } \mathrm{U}=2,50 \mathrm{~W} / \mathrm{m}^{2} \mathrm{~K} \\
\mathrm{Fs}=0,61\end{array}$ \\
\hline & $\begin{array}{l}\text { Inercia de } \\
\text { forjado }\end{array}$ & \multicolumn{2}{|c|}{$\begin{array}{l}\text { Losa de hormigón armado, } \mathrm{e}=0,3 \mathrm{Om} \\
\mathrm{m}=750 \mathrm{~kg} / \mathrm{m}^{2}\end{array}$} \\
\hline & $\begin{array}{l}\text { Inercia de } \\
\text { fachada }\end{array}$ & \multicolumn{2}{|c|}{$\begin{array}{l}\text { SATE (Sistema de aislamiento térmico } \\
\text { exterior) }\end{array}$} \\
\hline \multicolumn{4}{|c|}{ PROPUESTA BÁSICA + VENTILACIÓN (MV) } \\
\hline MV & Ventilación & \multicolumn{2}{|c|}{$\begin{array}{l}\text { Decreto francés 24/05/2006: } \\
0,3 \text { renov/h }\end{array}$} \\
\hline \multicolumn{4}{|c|}{ PROPUESTA BÁSICA + PROTECCIÓN SOLAR (MP) } \\
\hline \multirow[t]{2}{*}{ MP } & \multirow[t]{2}{*}{$\begin{array}{l}\text { Protección } \\
\text { solar }\end{array}$} & $\begin{array}{l}\text { Este } \\
\text { Oeste } \\
\text { Sur }\end{array}$ & $\begin{array}{l}\text { o \% de protección invierno y } \\
100 \% \text { verano }\end{array}$ \\
\hline & & Norte & o \% de protección \\
\hline
\end{tabular}

En la zona climática $\mathrm{B}$ 4, la importante radiación solar tiene una notable incidencia sobre la demanda energética de refrigeración. Por ello una tercera estrategia es la combinación de la propuesta básica (M) con un sistema de protec- ción solar móvil en los huecos consistente en una protección que impida en un $100 \%$ el paso de la radiación solar en verano y del o \% en invierno (MP) (Tabla 4). Finalmente se propone una estrategia de conjunto que reúne las tres opciones, modelo de propuesta básica, ventilación y protección solar (MVP).

Se realiza un análisis comparativo de cada estrategia combinada respecto del modelo base de la demanda anual de calefacción, refrigeración y total (Figura 2). Se observa que el modelo de propuesta básica $(\mathrm{M})$ en orientación este-oeste supone una importante reducción de demanda de hasta un $38 \%$ en demanda de calefacción y un $16 \%$ en demanda de refrigeración respecto del modelo base inicial. Sin embargo la demanda de calefacción de 17,02 kWh/m² es superior al valor máximo establecido por el DB HE 1 de 2013 de $15 \mathrm{kWh} / \mathrm{m}^{2}$. En cambio, en la orientación norte-sur la demanda de calefacción desciende a $14,5 \mathrm{kWh} / \mathrm{m}^{2}$, con un $36,5 \%$ de reducción de demanda total en dicha orientación respecto al modelo base inicial.

Las mejoras de protección solar introducidas en los modelos MP mantienen en ambas orientaciones la demanda de calefacción, no satisfaciendo por ello las exigencias mínimas del DB HE 2013 en la orientación este-oeste. Sin embargo suponen una importante mejora en la demanda de refrigeración, de hasta un $40 \%$ respecto del modelo base en la orientación este-oeste y del 23,5\% en la norte-sur.

La introducción de una reducción en la tasa de ventilación a $0,3 \mathrm{ren} / \mathrm{h}$ presente en los modelos MV suponen un importante descenso en las demandas respecto del modelo base, produciéndose reducciones de demanda total del $60 \%$ en la

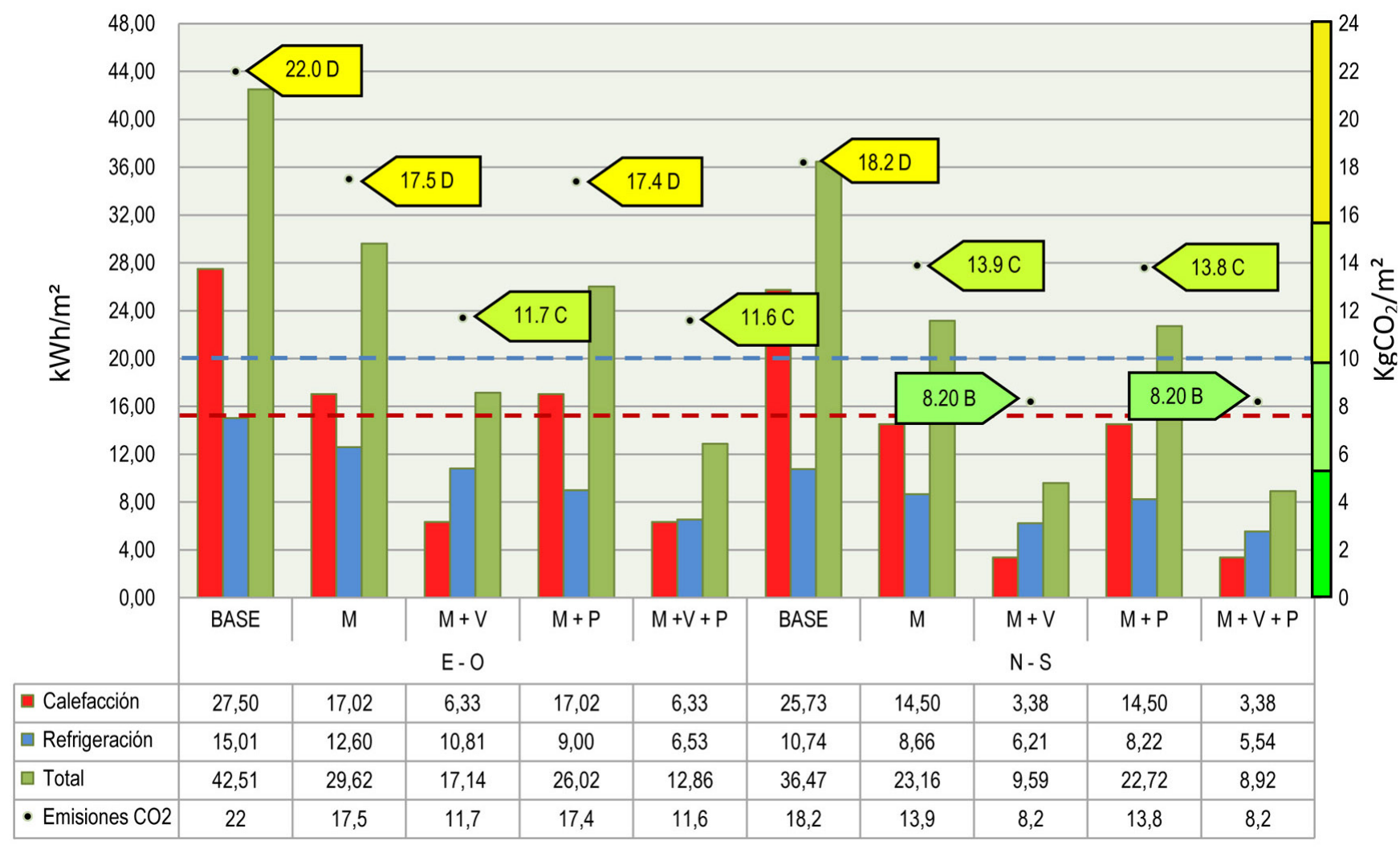

Figura 2. Calificación energética y evolución de las demandas anuales de calefacción, refrigeración y total en los modelos de propuesta referenciados a los límites de demanda del DB HE1 2013. 


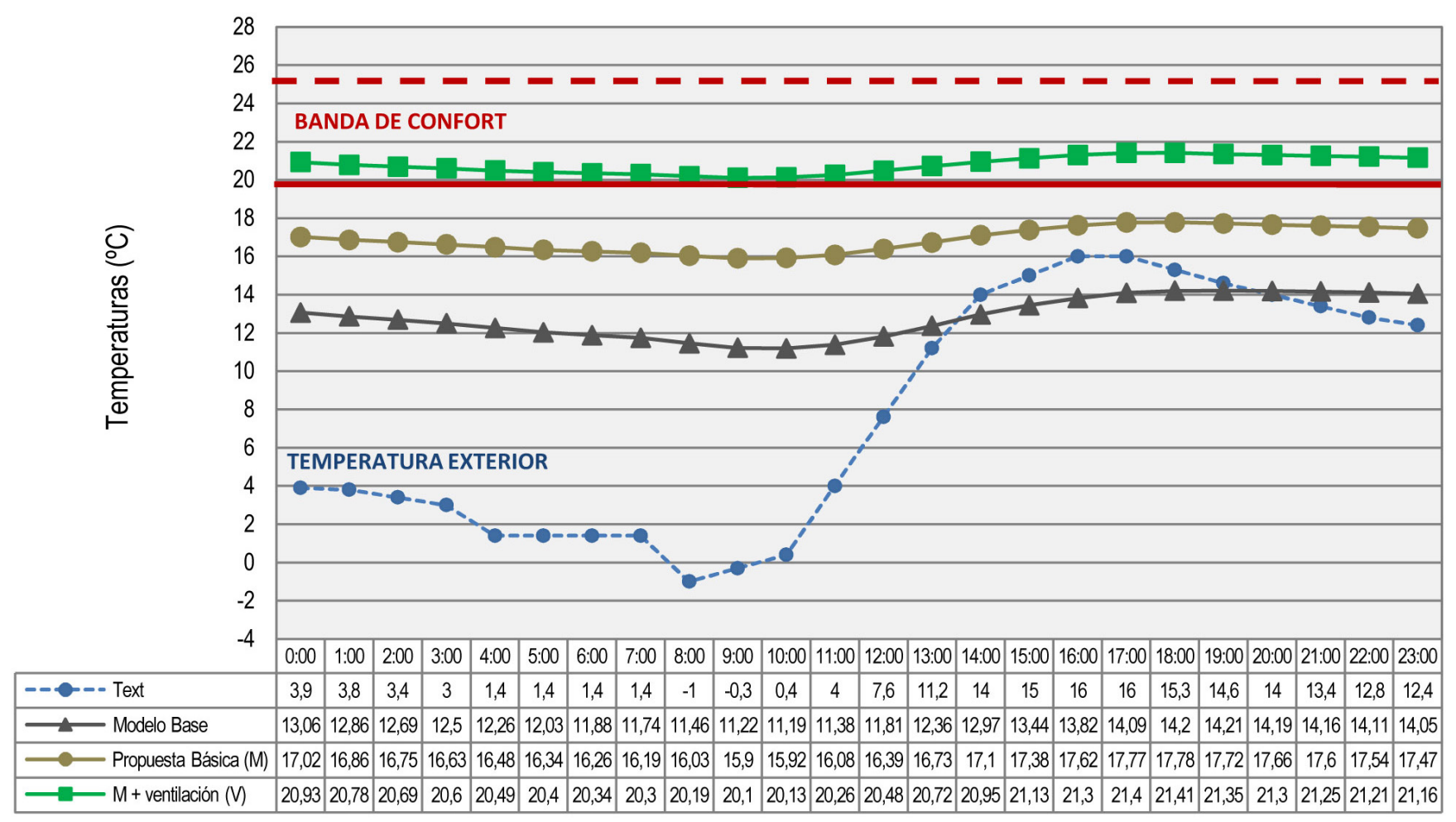

Figura 3. Gráfica de temperaturas en libre evolución en vivienda intermedia para el 16 de enero orientación norte-sur. Estrategia combinada MVP.

orientación este-oeste y del $74 \%$ en la orientación norte-sur, debido principalmente a una importante reducción de las demandas de calefacción.

La propuesta de combinación de la mejora de los elementos constructivos, ventilación y de protección solar (MVP) es la más efectiva, con bajos valores de demanda de calefacción, $6,33 \mathrm{kWh} / \mathrm{m}^{2}$ y refrigeración $6,53 \mathrm{kWh} / \mathrm{m}$ en orientación este-oeste, que disminuyen en la orientación norte-sur, tanto en calefacción, $3,38 \mathrm{kWh} / \mathrm{m}^{2}$, como en refrigeración, $5,54 \mathrm{kWh} / \mathrm{m}^{2}$.

Se realiza el estudio de las emisiones de $\mathrm{CO}_{2}$ del modelo base y de las diferentes propuestas de estrategias combinadas mediante la herramienta Calener VYP, para obtener la calificación energética del edificio. Se observan mejoras importantes de la calificación energética para las distintas propuestas (Figura 2). El modelo base presenta una calificación $\mathrm{D}$ que se mejora a una calificación $\mathrm{C}$ en la orientación norte-sur para el modelo de propuesta básica (M). La propuesta de ventilación (MV) consigue una calificación $\mathrm{C}$ en la orientación este-oeste, llegando a B en la orientación norte-sur. El modelo más cercano a la calificación A es la propuesta de mejora de los elementos constructivos, ventilación y de protección solar (MVP) en orientación norte-sur, con emisiones de $8,20 \mathrm{kgCO}_{2} / \mathrm{m}^{2}$, cercanas a los $5,5 \mathrm{kgCO}_{2} / \mathrm{m}^{2}$ de límite para obtener la calificación A.

Finalmente se realiza una valoración cualitativa en cuanto a las mejoras de confort interior debidas a las estrategias pasivas. Con la estrategia combinada (MVP) se observa, una situación de confort térmico durante todas las horas del día tipo de invierno, 16 de enero (Figura 3), mientras que en el día tipo de verano, 12 de julio (Figura 4), las temperaturas interiores son superiores a las de confort a lo largo de prácti- camente la totalidad del día con diferencias respecto a la temperatura de confort de hasta $2{ }^{\circ} \mathrm{C}$ a las 18:00, y prácticamente ajustadas a la banda de confort adaptativo durante las horas nocturnas debido a la ventilación nocturna de $4 \mathrm{ren} / \mathrm{h}$ definida por el DB HE1.

\section{CONCLUSIONES}

El camino hacia el Edificio de Energía Casi Nula obliga a replantear la construcción y diseño de los edificios, un cambio de modelo en las exigencias hacia el camino del Horizonte 2020. Tras el análisis de los resultados se ofrecen unas pautas de decisión sobre los factores más determinantes en la demanda energética en un bloque lineal entre medianeras de vivienda social en la zona climática $\mathrm{B} 4$, evitando soluciones únicas, y proponiendo ámbitos de decisiones adaptables a diferentes condiciones tipológicas y constructivas.

Frente a las exigencias del DB HE 2006, las actuales exigencias del DB HE 2013 suponen un enfoque más flexible, con mayor libertad para el proyectista, avanzando en el enfoque prestacional. Las necesarias mejoras de las prestaciones energéticas de la envolvente térmica deben avanzar respecto a los valores límite de referencia de los parámetros característicos del DB HE 2013, aumentando el espesor de aislamiento en elementos opacos; mejorando las prestaciones del hueco, principalmente mediante vidrios con mayor cámara o bajo emisivos; controlando la tasa de infiltración mediante la mejora mínima de las carpintería a clase 3 ; y acumulando y regulando las ganancias caloríficas mediante la inercia térmica, principalmente de los forjados con una masa del orden de $750 \mathrm{~kg} / \mathrm{m}^{2}$.

Llevados al límite aislamiento y sistemas constructivos, hay que trabajar con factores que dependen del diseño arquitec- 


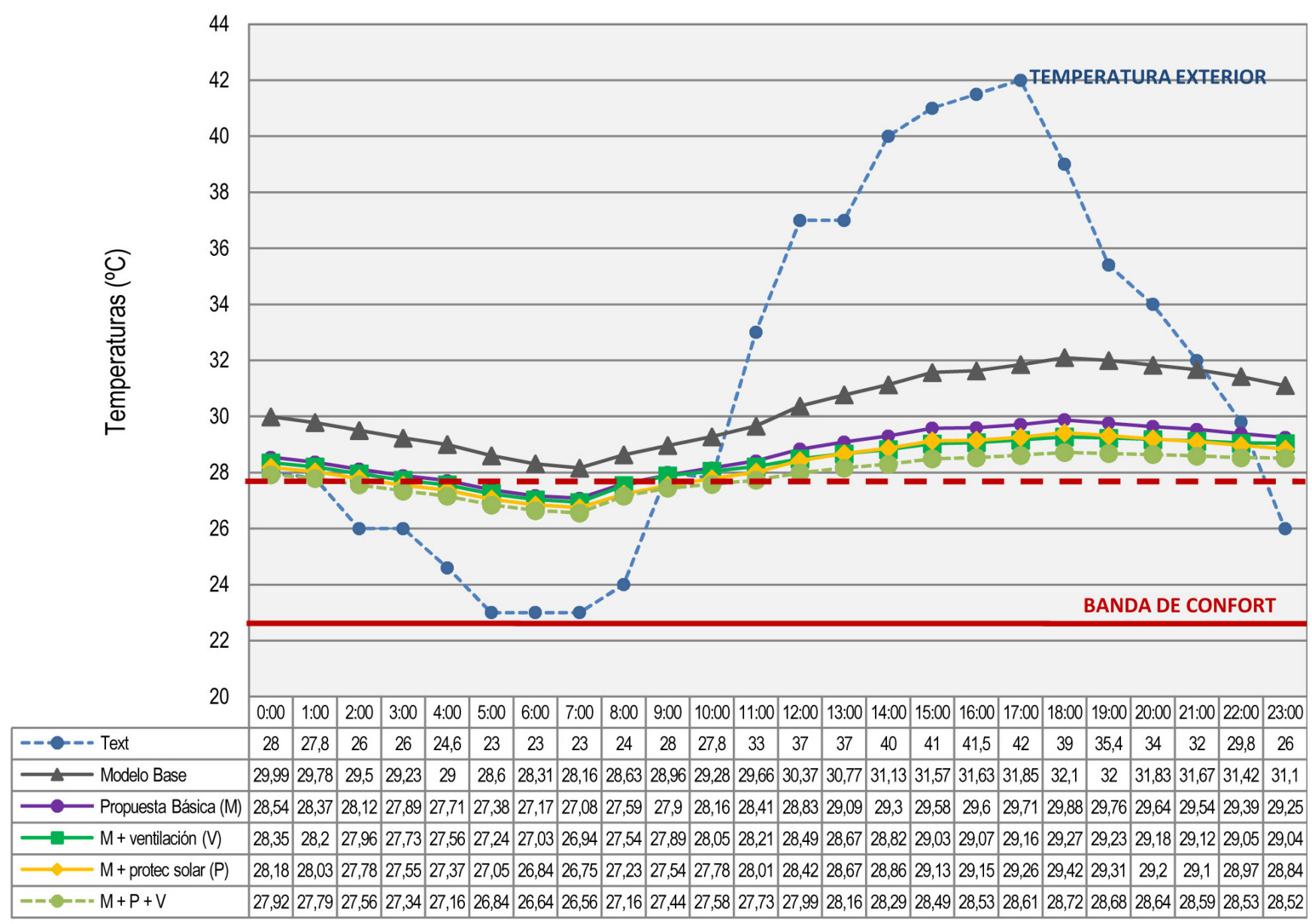

Figura 4. Gráfica de temperaturas en libre evolución en vivienda intermedia para el 12 de julio orientación norte-sur. Estrategia combinada MVP.

tónico y del urbanismo. La orientación del edificio es un instrumento muy eficaz para reducir la demanda, suponiendo la orientación norte-sur una mejora del orden del 8 al $16 \%$ frente a la este-oeste, siendo importante en ambas orientaciones ajustar el porcentaje de huecos a un valor no superior al $30 \%$. Del mismo modo la compacidad juega un papel importante en el balance energético, disminuyendo la demanda al aumentar ésta.

La tasa de ventilación es un factor decisivo en el balance energético de los edificios. La actual exigencia del DB HS3 de calidad del aire interior, basada en criterios de máxima ocupación, y su duplicidad, junto con la propuesta de sistemas de caudal constante implica un aumento de la demanda que dificulta los cumplimientos de las exigencias del DB HE1 2013. Se hace necesario afrontar la calidad del aire interior desde prestaciones reales, mediante el control de los niveles de concentración de $\mathrm{CO}_{2}$ o mediante sistemas de caudal variable.

La protección solar es un factor de gran relevancia para el control de la demanda de refrigeración del edificio, en una zona climática con una importante radiación solar. Los sistemas de protección solar deben favorecer la captación solar en invierno y el control solar en verano, principalmente en las fachadas más desfavorables, este y oeste.
La combinación de la mejora de las prestaciones energéticas de la envolvente térmica, de la tasa de ventilación y de la adecuada protección solar supone unas acciones de mejora energética de aplicación genérica a una edificación de vivienda social, que muestran la posibilidad de acceder a importantes reducciones de la demanda energética con soluciones «accesibles». Estas propuestas pueden reducir los valores de demanda de calefacción a 3-6 kWh/m² y de refrigeración a 5-6 kWh $/ \mathrm{m}^{2}$, lo que supone reducciones de demanda total del 70-75\% respecto un modelo de estricto cumplimiento del DB HE 2006. Además se obtiene una importante reducción de emisiones de $\mathrm{CO}_{2}$ lo que supone poder obtener con medios pasivos una calificación energética $\mathrm{B}$. El paso a la calificación A se obtendría con una mejora de la contribución solar, superior al mínimo exigido por el DB HE4, y con la incorporación de sistemas de climatización eficientes.

Frente a los objetivos cuantitativos regulados por la normativa, se incorpora un objetivo transversal cualitativo: el confort interior. Las soluciones de mejora pasivas propuestas permiten obtener una distribución de temperaturas operativas en el interior de las viviendas en libre evolución, muy cercanas a la banda de confort adaptativo durante gran parte del año, por lo que la utilización de sistemas activos de climatización resultaría necesaria puntualmente, excepto los días más calurosos del año, donde sería necesario refrigerar en las horas más calurosas. 


\section{REFERENCIAS}

(1) Presidencia de Gobierno. (1979, 22 de octubre). Real Decreto 2429/1979, de 6 de julio, por el que se aprueba la norma básica de edificación NBE-CT-79, sobre condiciones térmicas en los edificios. Boletín Oficial del Estado, n 253 , pp. 24524-24550. España.

(2) Ministerio de Vivienda. (2006, 28 de marzo). Real Decreto 314/2006 de 17 de marzo. Código Técnico de la Edificación. Boletín Oficial del Estado, $\mathrm{n}^{0} 74$, pp. 11816-11831.

(3) Ministerio de Fomento. (2013, 10 de septiembre). Orden FOM/1635/2013, de 10 de septiembre, por la que se actualiza el Documento Básico DB-HE «Ahorro de Energía», del Código Técnico de la Edificación. Boletín Oficial del Estado, $\mathrm{n}^{\circ} 219$, pp. 67137-67209.

(4) Garcia-Hooghuis, A., Neila, F.J. (2013). Modelos de transposición de las Directivas 2002/91/CE y 2010/31/UE "Energy Performance Building Directive" en los Estados miembros dela UE. Consecuencias e implicaciones. Informes de la Construcción, 65(531): 289-300, doi: http://dx.doi.org/10.3989/ic.12.017.

(5) Holl, M. (2010, 15-16 de noviembre). The new EPBD and its policy towards nearly zero energy buildings. En SBN Conference. París.

(6) Ábalos, I. (2008). La belleza termodinámica. Circo, 157.

(7) Balaras, C. A., Gaglia, A. A., Georgopoulou, E., Mirasgedis, S., Sarafidis, Y., Lalas, D. L. (2007). European residential buildings and empirical assessment of the Hellenic building stock, energy consumption, emissions and potential energy savings. Building and Environment, 42(3): 1298-1314 doi: http://dx.doi.org/10.1016/j.buildenv.2005.11.001.

(8) Jaber, S., Ajib, S. (2011). Optimum, technical and energy efficiency design of residential building in Mediterranean región. Energy and Buildings, 43(8): 1829-1834, doi: http://dx.doi.org/10.1016/j.enbuild.2011.03.024.

(9) Domínguez, S., Sendra, J. J., León, A.L., Esquivias, P. (2012). Towards energy demand reduction in social housing buildings: Envelope system optimization strategies. Energies, 5(7): 2263-2287, doi: http://dx.doi.org/10.3390/en5072263.

(10) Aste, N., Angelotti, A., Buzzetti, M. (2009). The influence of external walls thermal inertia on the energy performance of well insulated buildings. Energy \& Buildings, 41(11): 1181-1187, doi: http://dx.doi.org/10.1016/j.enbuild.2009.06.005.

(11) Al-Sanea, S.A., Zedan, M.F. (2012). Effect of thermal mass on performance of insulated building walls and the concept of energy savings potential. Applied Energy, 89(1): 430-442, doi: http://dx.doi.org/10.1016/j.apenergy.2011.08.oo9.

(12) Stazi, F., Bonfigli, C., Tomassoni, E., di Perna, C., Munafò, P. (2015). The effect of high thermal insulation on high thermal mass: Is the dynamic behavior of traditional envelopes in Mediterranean climates still possible? Energy and Buildings, 88: 367-383, doi: http://dx.doi.org/10.1016/j.enbuild.2014.11.056.

(13) León, A.L., Domínguez, S., Campano, M.A., Ramírez-Balas, C. (2012). Improving by solar protections of the Energy demand of multi dwelling units in Mediterranean climate. Energies, 5(9): 3398-3424, doi: http://dx.doi.org/10.3390/en5093398.

(14) Sendra, J. J., Domínguez, S., León, A. L. (2011). Proyecto Efficacia. Optimización Energética en la vivienda colectiva (pp. 1-140). Sevilla: Publicaciones Universidad de Sevilla.

(15) Santamouris, M., Kolokotsa, D. (2013). Passive cooling dissipation techniques for buildings and other structures: The state of the art. Energy and Buildings, 57: 74-94, doi: http://dx.doi.org/10.1016/j.enbuild.2012.11.002.

(16) Sendra, J.J., Domínguez, S., Bustamante, P., León, A.L. (2013). Energy intervention in the residential sector in the south of Spain: Current challenges. Informes de la Construcción, 65(532): 457-464, doi: http://dx.doi.org/10.3989/ic.13.074.

(17) Zero Carbon Hub. (2010). Closing the gap between designed and built performance. http://www.zerocarbonhub.org. London.

(18) Sunikka-Blank, M., Galvin, R. (2012). Introducing the prebound effect: the gap between performance and actual energy consumption. Building Research and Information, 40(3): 260-273, doi: http://dx.doi.org/10.1080/09613218.2012.69 0952.

(19) Sakka, A.; Santamouris, M.; Livada, I.; Nicol, F.; Wilson, M.(2012). On the thermal performance of low income housing during heat waves. Energy and Buildings, 49, 69-77. http://dx.doi.org/10.1016/j.enbuild.2012.01.023.

(20) Mihalakakou, G., Santamouris, M., Tsangrassoulis, A. (2002). On the energy consumption in residential buildings. Energy and Buildings, 34(7): 727-736, doi: http://dx.doi.org/10.1016/So378-7788(01)00137-2.

(21) Van Hoof, J., Hensen, L. M. (2007). Quantifying the relevance of adaptive thermal comfort models in moderate thermal climate zones. Building and Environment, 42(1): 156-170, doi: http://dx.doi.org/10.1016/j.buildenv.2005.08.023.

(22) Ferrari, S., Zanotto, V. (2012). Adaptive Comfort: analysis and application of the main indices. Building and Environment, 49: 25-32, doi: http://dx.doi.org/10.1016/j.buildenv.2011.08.022.

(23) García-Navarro, J., González-Díaz, M. J., Valdivieso, M. (2014). «Estudio Precost\&e»: evaluación de los costes constructivos y consumos energéticos derivados de la calificación energética en un edificio de viviendas situado en Madrid. Informes de la Construcción, 66(535): e026. doi: http://dx.doi.org/10.3989/ic.13.052.

(24) Ministerio de Fomento. (2013). Report on Cost Optimal Calculations and Comparison with the current and future Energy Performance Requeriments of Buildindings in Spain. Madrid.

(25) IDAE. (2009). Condiciones de Aceptación de procedimientos alternativos a LIDER y CALENER. Madrid: Instituto para la Diversificación y Ahorro de la Energía, Ministerio de Industria, Turismo y Comercio.

(26) DesignBuilder versión 2.4.2.026. http://www.designbuilder.co.uk/

(27) Ruá, M. J., López-Mesa, B. (2012). Certificación energética de edificios en España y sus implicaciones económicas. Informes de la Construcción, 64(527): 307-318, doi: http://dx.doi.org/10.3989/ic.11.028.

(28) Legifrance. (2006, 25 de mayo). Arrêté du 24 mai 2006 relatif aux caractéristiques thermiques des bâtiments nouveaux et des parties nouvelles de bâtiments. $J O R F, \mathrm{n}^{\circ} 121$, Article 20. 